\title{
Hiperión como novela epistolar
}

\author{
Hyperion as an epistolary novel
}

\section{Hyperion como romance epistolar}

\author{
iD Juan Pau \\ Universidade de Buenos Aires (UBA), Cidade Autônoma de Buenos Aires, Argentina. \\ E-mail: juanrobertopau@hotmail.com \\ Mariela Ferrari \\ Universidade de Buenos Aires (UBA), Cidade Autônoma de Buenos Aires, Argentina. \\ E-mail: mariela_c_ferrari@hotmail.com
}

\begin{abstract}
Resumen: El objetivo del presente artículo es abordar la interrelación entre el género epistolar y el Bildungsroman en Hiperión, de Friedrich Hölderlin. Sus características como novela epistolar son congruentes con sus características como Bildungsroman, por lo que se encuentra justificado el género epistolar. De tal forma, el elemento de intimidad, la necesidad de un interlocutor y el tratamiento fragmentario del tiempo propios de la novela epistolar son funcionales a la formación del protagonista y del lector y al manejo temporal inherentes al Bildungsroman, respectivamente. Se analizarán ambos géneros (teórica y específicamente en la novela) y las mencionadas características en Hiperión.
\end{abstract}

Palabras clave: Hölderlin. Cartas. Bildungsroman.

\begin{abstract}
The goal of this article is to address the interrelation between the epistolary genre and the Bildungsroman in Hyperion, by Friedrich Hölderlin. Its features as an epistolary novel are consistent with its characteristics as a Bildungsroman, so the epistolary genre is justified. Thus, the element of intimacy, the need for an interlocutor, and the fragmentary treatment of time proper to epistolary novels are functional to the formation of the protagonist and the reader and to the handling
\end{abstract}


of time inherent in Bildungsromane, respectively. This article discusses both genres (theoretically and specifically in the novel) and the abovementioned features in Hyperion.

Keywords: Hölderlin. Letters. Bildungsroman.

Resumo: O objetivo deste artigo é abordar a interrelação entre o gênero epistolar e o Bildungsroman em Hyperion, de Friedrich Hölderlin. Suas características como romance epistolar são congruentes com suas características como Bildungsroman, portanto, o gênero epistolar é justificado. Assim, o elemento de intimidade, a necessidade de um interlocutor e o tratamento fragmentário do tempo próprios do romance epistolar são funcionais à formação do protagonista e do leitor e ao manejo do tempo inerente ao Bildungsroman, respectivamente. Tanto os dois gêneros serão analisados (teórica e especificamente no romance) quanto as características mencionadas em Hyperion.

Palavras-chave: Hölderlin. Cartas. Bildungsroman.

Submetido em 11 de setembro de 2020.

Aceito em 06 de outubro de 2020.

Publicado em 01de fevereiro de 2021. 


\section{Introducción}

Son frecuentes los debates académicos y críticos sobre la novela Hiperión o el eremita en Grecia (Hyperion, oder der Eremit in Griechenland), de Johann Christian Friedrich Hölderlin (17701843), en especial respecto de su pertenencia al género conocido como Bildungsroman (NAUEN, 1971, p. 56; DILTHEY, 2016, p. 353). A su vez, este género ha suscitado otros debates, más amplios aún, que incluso han llegado a cuestionar su existencia; como afirma Redfield: "it is uncertain whether this genre exists to be described in the first place" (1996, p. 40). No tan frecuentes han sido los debates en torno a la pertenencia de la obra al género de la novela epistolar. Por el contrario, mientras se menciona al pasar que Hiperión es una novela epistolar, sin mayor análisis, ${ }^{1}$ también se pone en duda la eficacia de la forma elegida y se la tacha de "injustificada". ${ }^{2}$ En el presente trabajo, nos proponemos demostrar que la forma epistolar en la novela no solamente está justificada, sino que resulta esencial, pues sus características son funcionales al Bildungsroman. Esto significa que las características de ambos géneros se complementan, combinan y potencian con el objeto de producir sentido en la novela. Es decir, cada una de las características que analizaremos encuentra su correlato en una característica del otro género que le resulta afín: más precisamente, el elemento de intimidad, la necesidad de un interlocutor y el tratamiento fragmentario del tiempo propios de la novela epistolar son funcionales a la Bildung del protagonista, a la del lector y al manejo temporal del Bildungsroman, respectivamente. Así pues, en la sección $2 \mathrm{~A}$ formularemos algunos comentarios sobre el Bildungsroman, en la sección 2B analizaremos con mayor detalle a Hiperión como novela de este tipo según las características mencionadas, en la sección 3A abordaremos brevemente la novela epistolar y en la sección 3B nos ocuparemos de Hiperión como

1 En palabras de Hoffmeister, constituye una "epistolary novel of a nostalgic retrospective to the golden age" (2004, p. 84). 2 Así, Silz se pregunta "is it possible to justify the letter form [...]?", y contesta: "I do not believe it is" (1969, p. 23). 
novela epistolar según los aspectos enumerados. En la conclusión, por último, retomaremos someramente estas cuestiones.

\section{El Bildungsroman e Hiperión}

\section{A. El Bildungsroman}

Para abordar el Bildungsroman, conviene tener presentes algunas nociones básicas sobre la Bildung, puesto que estamos en presencia de dos vocablos muy utilizados, mas no siempre con la rigurosidad correspondiente. Como sostiene Sammons, "the defining terminologies of literary criticism and history chronically generate problems" (1991, p. 26), por lo cual debemos ser cuidadosos con su uso. Además, según afirma Koval, "la noción de Bildung es muy compleja: cambia de un autor a otro e, incluso, de un texto a otro del mismo autor" (2018, p. 33). Lo que debe tenerse también en mente al momento de abordarla es que "Bildung is an historical concept that can be located in the Age of Goethe" (COCALIS, 1978, p. 407), lo cual nos sitúa en la época y el lugar de Hiperión.

Variados son los conceptos a los que se ha asimilado históricamente la noción de Bildung, tales como educación, desarrollo, cultivación, aprendizaje, entre otros. No obstante, ninguno logra reflejar cabalmente sus componentes semánticos. No apunta a cuestiones de enseñanza formal, tampoco a la inteligencia del individuo, ni al talento personal. En palabras de Horlacher, "Bildung es prácticamente intraducible a cualquier otro idioma" (2014, p. 36). Sin embargo, un elemento en el que coinciden varios autores es el de la formación personal, o autoformación, como aproximación al concepto bajo análisis.

Así pues, según Jost, "formation does not currently have the meaning of Bildung, a meaning perfectly rendered by the French formation and its philological equivalents in other Romance languages. Sich bilden means to give one's mind, one's character, and one's personality their final shape" (2014, p. 126; énfasis en el 
original). Por su parte, Horlacher manifiesta que "Bildung es el autocultivo interior que depende del exterior, pero que es mucho más noble que el mundo exterior y apunta a una realización armónica de la totalidad interna" (2014, p. 40). Finalmente, Mahoney señala que "in contrast to the concept of education, however, Bildung was supposed not just to come from outside mentors but also to represent a process of self-development" (1991, p. 109). ${ }^{3}$ A los efectos del presente trabajo, optaremos por el término formación o autoformación o utilizaremos el préstamo Bildung para referirnos a este concepto.

Con respecto al Bildungsroman, inicialmente, podríamos definirlo como novela de formación. ${ }^{4}$ Sin embargo, esta definición de por sí no resulta demasiado útil a los efectos analíticos, pues deja muchos interrogantes sin resolver. Se suele tomar como modelo del género Los años de aprendizaje de Wilhelm Meister (Wilhelm Meisters Lehrjahre, publicada en 1795 por Johann Wolfgang von Goethe), lo cual de todas maneras no impide poner en duda la existencia del género e incluso la pertenencia de dicha novela al género que supuestamente inauguró. Según Redfield, no existe certeza siquiera sobre la existencia del género (1996, p. 40) y, en palabras de Sammons, "even the identification of the archetypal text, Goethe's Wilhelm Meister, as a Bildungsroman has been challenged" (1991, p. 32).

Sin perjuicio de las dificultades y limitaciones señaladas, el Bildungsroman implica un cambio y un crecimiento (una autoformación, como consignamos más arriba) que se produce a lo largo del tiempo en la novela (género literario adecuado al efecto, dada su extensión), no siempre en íntima relación con los hechos narrados. Es decir, el resultado final de la formación en cuestión no siempre es relevante o determinante para definir si estamos ante un Bildungsroman. De esta manera, Sammons afirma que "it does not much matter whether the process of Bildung succeeds or

\footnotetext{
3 Desde luego, este es tan solo uno de los elementos del concepto de Bildung y abordarlo en su totalidad excedería el alcance de este trabajo. Koval (2018, p. 33s.) realiza un análisis pormenorizado de la cuestión.

4 Al igual que la noción de Bildung, el Bildungsroman obedece a circunstancias históricas específicas, lo cual debería llevarnos a evitar utilizarlo para cualquier tipo de novela. Como sostiene Swales, "the Bildungsroman was born [...] in specific historical circumstances, in a demonstrable interlocking of theory and praxis. It is a novel form recognizably animated by the Humanitätsideal of late eighteenth-century Germany" (1991, p. 49).
} 
fails, whether the protagonist achieves an accommodation with life and society or not" (1991, p. 41). Por el contrario, lo que importa es el camino recorrido, durante el cual se produce la Bildung. En tal sentido, Swales manifiesta que "the novel, then, is written for the sake of the journey and not for the sake of the happy ending which that journey seems to promise" (1991, p. 53).

\section{B. Hiperión como Bildungsroman}

Como indicamos más arriba, es objeto de debate la pertenencia de Hiperión $n^{5}$ al género del Bildungsroman. Silz cuestiona de modo enfático dicha pertenencia, ya que asevera que, a los efectos de su carácter de héroe en una novela de desarrollo, "Hyperion seems to have more faults than he needs for such purpose, and the final stage of his 'improvement' is not entirely clear" (1969, p. 58) y que, a raíz de la forma epistolar de la novela, "it is seldom demonstrable which ideas he had at the time reported and which he has evolved since" (1969, p. 19). De esta forma, Silz afirma que es contradictorio el uso de cartas de esta forma, pues se utilizan como memorias acompañadas por comentarios del presente, cuando en realidad deberían ser vehículos de comunicación directa e inmediata (1969, p. 18). Asimismo, sostiene que la novela es una canción de amor consagrada a los tres amores del poeta, la naturaleza, Grecia y Diotima, y que no estamos ante una novela de desarrollo, sino ante un autorretrato subjetivo y lírico (1969, p. 58). No obstante, creemos que estamos ante un Bildungsroman y que la forma

\footnotetext{
5 La novela (que había tenido 4 versiones previas: el Hyperion-Segment en 1793, el Fragment von Hyperion en la revista Thalia en 1794, la "versión métrica" en 1795 y el Hyperions Jugend en 1795) fue publicada en dos volúmenes, en 1797 y 1799 , cada uno de los cuales consta de dos libros (libro 1 del primer volumen: 11 cartas; libro 2 del primer volumen: 19 cartas; libro 1 del segundo volumen: 22 cartas; libro 2 del segundo volumen: 12 cartas). En su mayoría las escribe Hiperión, griego, a su amigo Belarmino, alemán, del cual no tenemos cartas. Casi todas son escritas con posterioridad a los hechos, pero algunas hacia el final (entre Hiperión y su amada, Diotima, y entre Hiperión y su amigo Notara) son transcriptas o adjuntadas para que las vea Belarmino, lo cual nos permite acceder a la versión de los hechos narrados mientras se estaban produciendo, cosa que no sucede con las demás cartas, que narran hechos mucho después de ocurridos. Hiperión rememora su experiencia en sus viajes a su Grecia natal. El relato está situado en 1770, en ocasión de las luchas entre turcos y griegos. Durante su periplo, Hiperión conoce a Adamas, un maestro que le transmitirá una serie de conocimientos, a Alabanda, con el cual forjará los proyectos de liberación de Grecia, y a Diotima, de la cual se enamorará. En los tres casos las relaciones se rompen por distintos motivos, con el agravante de que, con Alabanda, se produce un reencuentro y una nueva decepción, al fracasar los planes de liberación de Grecia de la opresión otomana. Hiperión sufre una terrible desilusión y opta por morir, lo cual llega a conocimiento de Diotima, que decide hacer lo mismo. Sin embargo, Hiperión también fracasa en su proyecto de morir, lo cual lo deja solo y lo incentiva a escribirle a Belarmino, tras su pedido.
} 
epistolar es fundamental en ese sentido, como analizaremos a continuación.

Como se observa a lo largo de la obra, Hiperión rechaza todo viso de educación formal ligado a instituciones, dado que, desde el principio, afirma "¡ojalá no hubiera ido nunca a vuestras escuelas! La ciencia [...] es la que me ha estropeado todo"; agrega que "en vuestras escuelas es donde me volví tan razonable" y que "he sido así expulsado del jardín de la naturaleza" (HÖLDERLIN, 1996, p. 26). ${ }^{6}$ Valora también la etapa de la niñez como estadio de la vida en el que el ser humano cuenta con la mayor libertad posible: "La coerción de la ley y del destino no le andan manoseando; en el niño solo hay libertad" (p. 27). Hiperión, como muchos héroes del Bildungsroman, comienza su periplo abandonando la casa paterna, lo cual es visto por él como su "nacimiento" y le permite ocuparse de su "formación" (p. 39).

Las cuestiones estéticas y naturales tienen preponderancia en la Bildung hiperioniana. Hiperión afirma que "en el niño debe vivir una naturaleza completa antes de que vaya a la escuela" (p. 112), que "de la pura inteligencia no brotó nunca nada inteligible (...) Sin belleza de espíritu, la inteligencia es un siervo artesano" (p. 117) y que los que buscan "lo mejor en la profundidad del saber, en el tumulto del comercio, en la oscuridad del pasado, en el laberinto del futuro, en las tumbas o más arriba de las estrellas" deben saber que "su nombre es belleza" (p. 80).

Si bien podemos afirmar, al igual que Fuerst, que, en Hiperión, el "educational content is far from being as conspicuous as Hölderlin had, at times, intended it to be" (1946, p. 341), debemos también poner de manifiesto que el acto de escritura constituye un hecho relevante de por sí. ${ }^{7}$ En otras palabras, la cuestión de la autoformación se encuentra íntimamente vinculada con el acto de narrar. En una lectura frecuentemente mencionada, Mahoney sostiene que "Ryan traces Hyperion's development through the

6 Todas las citas de la obra pertenecen a esta edición.

7 Otros críticos, como Stephenson, ven el proceso de formación de Hiperión de manera más lineal: “Hölderlin's Hyperion is divided into four books, each of which deals with a distinct phase of Hyperion's interactive education: his formative childhood experience in the first book; in the second, his transformation through love; active experimentation in the third; and, in the fourth, reflective coming-to-terms with death" (2005, p. 218). 
narration of his life story: it is not his calamitous life history [...] but his retelling of these events that transforms him into a poet" (1991, p. 101s; énfasis en el original). La formación se produce a través de la transmisión o la expresión escrita de los hechos y no a raíz de las vivencias en sí. Por su parte, Dilthey señala que "Hiperión [...] pasó del curso de los acontecimientos a la comprensión de su significado" y que "la sucesión del curso de vida que representa la serie de cartas se halla articulada por la conciencia coherente de una mirada retrospectiva" (2016, p. 363). Al tener que organizar mentalmente y poner por escrito los hechos vividos, Hiperión los comprende.

La narración de su historia de vida y su transformación en escritor (su contacto con el arte) le permiten a Hiperión experimentar la Bildung a la que nos hemos referido. En tal sentido, reviste fundamental importancia para Hiperión el hecho de narrar: pone de relieve a los narradores, "los únicos a los que a veces escuchaba", considerados por él "archivos vivientes de nombres de ciudades y países extranjeros" (p. 42), tras contar una historia a los presentes afirma que "yo relaté la historia y al final todos nos sentíamos más fuertes y elevados" (p. 137) y afirma que cuenta "todo esto" porque "cada alentar de la vida sigue siendo valioso para nuestro corazón, porque todas las metamorfosis de la naturaleza pura pertenecen también a su belleza". De esta forma, pone de manifiesto que la narración tiene un efecto, tanto en el narrador como en sus oyentes/lectores. Por otra parte, es el mismo Hiperión el que aprecia su cambio con el transcurso de la narración: "incluso en mis cartas podrás apreciar cómo mi alma se vuelve cada día más y más tranquila" (p. 141s.).

Además, Hiperión llega a comprender la importancia del dolor: "el bienestar sin sufrimiento es sueño, y sin muerte no hay vida. ¿Querrás ser eternamente como un niño y dormitar como la nada? [...] ¿No recorrer la escala de los perfeccionamientos? ¡Sí, sí, el dolor es digno de habitar en el corazón [...] solo él conduce de un placer a otro!" (p. 200). Finalmente, logra desprenderse de sus 
añoradas ideas de juventud: "las ideas de mi juventud, que tuve por grandes, ya no me sirven" (p. 202).

También es objeto de debate si la Bildung es la del protagonista o la del lector. En Hiperión, son varios los pasajes en los cuales los lectores son interpelados más o menos directamente, como el prefacio y la penúltima carta (analizados más detalladamente en la sección siguiente). Asimismo, también se ha señalado el doble destinatario de la Bildung en la novela: no solamente su protagonista, sino los lectores. Fuerst apunta que "the intention is clear, not only to show the (self-)education of the hero, but also to educate" (1946, p. 344). Por su parte, Mahoney, en una síntesis de tres lecturas posibles y simultáneas, manifiesta que "Hyperion represents not only the development of a character (Dilthey) and the process of maturation of a poet (Ryan), but also the envisioned formation of a reader" (1991, p. 103). Es decir, para estos críticos, el lector debe alcanzar el punto de vista que logró el narrador a través de sus cartas.

El factor temporal constituye también un elemento importante en la novela, tanto en relación con su carácter de Bildungsroman ${ }^{8}$ como en relación con su carácter epistolar, como analizaremos en la sección siguiente. En este sentido, es útil la descripción que hace Miles del confesor, como contraposición al pícaro: "the confessor (...) experiences time in a radically different manner: as being complex, multilayered, and psychological (in the sense of being nonchronological); the self of the confessor does not exist a priori, but must be recollected" (1974, p. 980; énfasis en el original). Esta descripción es adecuada para Hiperión, dado que la complejidad y el factor psicológico juegan un papel importante en la cuestión temporal de la novela, pues Hiperión narra experiencias acaecidas a lo largo de toda su vida, pero no de manera lineal, sino fragmentaria. Como afirma Silz, "the close of the novel sets forth views which the hero has renounced in favor of more mature and valid insights stated a number of pages earlier, in the narrator's present, while his closing statement belongs to his abandoned

8 Como indica Koval, "a la formación le es inherente un contenido temporal" (2018, p. 33); según Jost, "the Bildungsroman [...] is also a psychological novel. In psychology the time factor is essential" (2014, p. 141). 
past" (1969, p. 19). Es decir, si bien la narración no sigue un estricto orden cronológico ni lineal, creemos que Hiperión sí pudo experimentar la Bildung y formarse, en este caso como narrador o artista, según lo consignado anteriormente.

Hemos analizado someramente cómo se produce la Bildung en Hiperión y abordado algunos elementos que nos indican la pertenencia de la novela al género. ${ }^{9}$ La autoformación del protagonista, a través de su crecimiento como narrador que adopta una postura cada vez más calma y pensante a lo largo del relato, es uno de esos elementos. Asimismo, el tratamiento fragmentario de la cuestión temporal, esencial para el Bildungsroman, tiene características particulares que obedecen a las vicisitudes de la formación del protagonista. Por último, la formación del lector también es un elemento propio del Bildungsroman en general y relevante en nuestra novela, dadas las interpelaciones a los lectores (principalmente el pueblo alemán) a las que recurre el narrador en reiteradas oportunidades. A continuación analizaremos más detalladamente cómo se vinculan estos tres elementos con las características de la novela epistolar y por qué dicho género reviste fundamental importancia en la novela.

\section{La novela epistolar e Hiperión}

\section{A. La novela epistolar}

La novela epistolar, muy popular en el siglo XVIII, implica contar una historia a través de cartas. Una distinción que se ha apuntado (FRAANJE, 2001) es la que existe entre las novelas en que las cartas son redactadas en su totalidad después de todos los hechos narrados y las novelas en que las cartas se redactan a medida que se producen los hechos (es decir, cuando termina una carta, todavía no sucedió lo que se narrará en la siguiente). Esta

\footnotetext{
9 Desde luego, la pertenencia al género en sí no nos dice mucho. Como sostiene Fuerst, "the interpreter who applies the term to a novel does not do more than construct a scaffolding, a very temporary and auxiliary structure, in order to get closer to the only lasting reality, the individual work of art" (1946, p. 340). De todos modos, creemos pertinente dejarla asentada a los efectos de señalar la íntima relación entre las características de Hiperión como Bildungsroman y como novela epistolar.
} 
distinción debe tenerse en cuenta a la hora de analizar Hiperión porque, si bien la mayoría de las cartas fueron redactadas tras la conclusión de los hechos narrados, se intercalan algunas narradas a medida que van sucediendo los acontecimientos, lo cual habilita un análisis diferente, particularmente en lo que respecta al cuestión temporal, como veremos en la sección siguiente.

Como afirma Spang, "la novela epistolar [se] desarrolla en relación con [...] el afán de autoanálisis y cierto 'confidencialismo' y 'confesionalismo' literarios tan representativos del Prerromanticismo europeo" (2000, p. 640). La carta es un elemento literario que suscita interés en amplios sectores de la población europea. En efecto, "the letter poses as a real-life document (and it certainly did in the eighteenth century)" (ALTMAN, 1982, p. 161). Por añadidura, "as a reflection of self [...], the letter connotes privacy and intimacy; yet as a document addressed to another, the letter reflects the need for an audience, an audience that may suddenly expand when that document is confiscated, shared, or published" (ALTMAN, 1982, p. 186s.). Estas características resultan fundamentales para un Bildungsroman porque la interioridad del protagonista se resalta en el carácter íntimo de la carta y su transmisibilidad responde a las necesidades de la novela de ser atractiva para el lector y se vincula con la Bildung del lector, a la cual nos hemos referido anteriormente. Otros elementos importantes para la Bildung y propios de la novela epistolar son el psicológico ${ }^{10}$ y el tiempo de reflexión que tiene el autor/narrador de las cartas, ${ }^{11}$ un tiempo que, como hemos observado en la sección anterior, resulta fundamental para la formación de Hiperión.

Un párrafo aparte merece el receptor de las cartas. Como en cualquier intercambio epistolar, el remitente de la carta necesita tener una persona con la suficiente cercanía o confianza para contarle sus pensamientos más íntimos. ${ }^{12}$ Además, la carta en tanto género discursivo exige una respuesta lato sensu por parte

10 "[S]tress will be thrown off events and onto psychology"; "confidential exchange is motivated by psychological need" (ALTMAN, 1982, p. 21 у р. 61).

11 "'TThe letter [...] can also 'give one a chance to consider -think matters over, and reach a more balanced view"' (ALTMAN, 1982, p. 38).

12 "[T]he confidant who inspires, wins, or loses trust is an essential figure in epistolary literature" (ALTMAN, 1982, p. 50). 
del destinatario. ${ }^{13}$ Esta característica también es funcional al Bildungsroman, sobre todo, si se tiene presente la Bildung del lector, dado que el autor de toda novela epistolar tiene en mente tanto al lector interno de cada una de las cartas como al lector externo de su obra. En tal sentido, es esperable que la carta tenga por objeto producir efectos o suscitar una respuesta en ambos: "the epistolary form is unique in making the reader (narratee) almost as important an agent in the narrative as the writer (narrator)" (ALTMAN, 1982, p. 88).

\section{B. Hiperión como novela epistolar}

El carácter de Hiperión como novela epistolar es central para analizar los elementos mencionados anteriormente $y$, a su vez, relacionarlos con los del Bildungsroman. A diferencia de Silz, quien no cree que sea posible justificar la forma epistolar (SILZ, 1969, p. 23), aquí consideramos que esta se encuentra totalmente justificada y es funcional y esencial a la producción de significado en la obra. Las características de ambos géneros se complementan, combinan y potencian. Cada una de las características analizadas respecto del Bildungsroman encuentra su correlato en una característica del género epistolar que le resulta afín. Ello obedece, en parte, a lo que afirma Mahoney, para el que Hölderlin "returned to his original plan for an epistolary novel: by virtue of the long tradition of this genre in the eighteenth century, readers would [...] view themselves as the recipients of Hyperion's letters and [...] follow with active concern the narration of his story" (1991, p. 102), $y$, también, a los motivos que analizaremos a continuación.

El elemento de intimidad propio de la novela epistolar se encuentra presente a lo largo de toda la novela, pues Hiperión, en ocasión de su intercambio con Belarmino, narra sus sentimientos y sensaciones durante los acontecimientos descriptos. Como ya lo hemos señalado, este elemento reviste una importancia

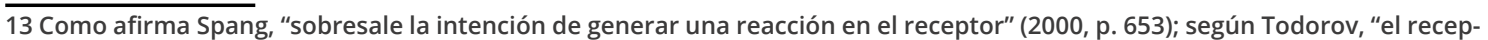
tor mismo se encuentra en estrecha relación con el enunciado" (1971, p. 31). 
fundamental en la Bildung y es funcional a la formación del protagonista. Hiperión le cuenta a Belarmino que "muchas cosas han cambiado en mi mirada y ahora hay en mí suficiente paz como para permanecer tranquilo ante cualquier aspecto de la vida humana" (p. 141), en uno de los tantos pasajes que nos muestra cómo la comunicación epistolar le permite al protagonista expresar su ser íntimo más fielmente.

Asimismo, no podemos pasar por alto el prefacio de la novela, que podría tomarse como una carta más o, al menos, una carta al lector. Si bien no tiene formato de carta, sí hay referencias al lector en relación con el libro que está a punto de iniciar. El narrador sostiene, respecto de los lectores preocupados en exceso por la moraleja y de los que lo tomen demasiado a la ligera, que "ni unos ni otros lo comprenderán" (p. 21) y, luego, afirma haberse avergonzado "de haber sobrevalorado de forma tan excesiva el posible juicio del público". Según Mahoney, estás palabras reflejan "the author's skepticism regarding his own chances for success among the German reading public" (1991, p. 113). El mismo autor afirma, para proporcionar otra interpretación, que "Ulrich Gaier understands these prefatory words as a challenge to Hyperion's readers; if they pass the test, they, like Bellarmin, are no longer to be counted among those Germans against whom Hyperion inveighs so fiercely near the conclusion of the novel" (p. 113). Es decir, la referencia y la interpelación a los lectores son directas y explícitas, por lo cual no las podemos pasar por alto y las debemos analizar a la luz del tipo de novela del que forman parte.

De modo análogo, debemos tener presentes otras dos referencias al público lector: hacia la mitad de la novela, Hiperión le dice a Belarmino "solo a ti, Belarmino, solo a un alma pura [...] se lo cuento [...]. No quiero echar mis perlas a la masa estúpida" (p. 100) y, al final, en la penúltima carta, tenemos la cáustica diatriba dirigida al pueblo alemán: "bárbaros desde tiempos remotos"; “las virtudes de los alemanes son brillantes maldades"; "no hay en este pueblo nada sagrado que no haya sido profanado y rebajado", etc. (p. 204-206). Como se observa en las críticas al principio, en el 
medio y al final de la novela, aquí no se produce ningún cambio, desarrollo ni modificación del narrador/Hiperión. La opinión respecto del público es siempre la misma. Si bien estamos de acuerdo con la tesis del desafío consignada por Mahoney respecto de la primera parte, creemos que la carta del final, al ser tan virulenta, no tiene otro objeto que describir lo más crudamente posible la realidad alemana. Quizás por eso es que, como afirma Mahoney, "the almost total neglect of Hyperion among Hölderlin's contemporaries confirmed his skepticism" (1991, p. 113s.).

Respecto del factor temporal en la novela epistolar, a diferencia de lo que sostienen algunos críticos (SILZ, 1969, p. 18; FRANZEL, 2009, p. 160), ${ }^{14}$ Hiperión no es en su totalidad una novela en la que se comienzan a escribir las cartas después de concluidos todos los hechos narrados (ver primer párrafo de sección 3A). Hacia el final del primer libro del segundo volumen, se reproduce el intercambio entre Hiperión y Diotima: "[T]e copio ahora las cartas que Diotima y yo nos escribimos tras mi despedida [...]. Son la imagen más cálida de aquellos días de mi vida" (p. 142); luego se transcriben intercambios con Notara, lo cual agrega un nivel más a la complejidad temporal de la novela: a las brechas temporales entre lectura y escritura de cualquier obra literaria se suman las brechas temporales ficticias que se producen entre la escritura y lectura de cada carta y, en este caso, un tercer "nivel" de brechas temporales entre escritura, lectura, respuesta y lectura de las cartas entre Hiperión y Diotima e Hiperión y Notara. Hacia el final de la obra, Hiperión transcribe las cartas de Diotima en sus cartas a Belarmino (o sea, no le envía copias a su amigo, sino que incorpora la voz de Diotima en sus propias cartas), lo cual también suma complejidad. ${ }^{15}$

Si bien las cartas no tienen fechas que nos puedan indicar a lo largo de cuánto tiempo se escribieron, el hecho de estar ante una gran cantidad de cartas es una clara muestra de que ese plazo no

\footnotetext{
14 Silz afirma que "there is no inherent reason for cutting off a past story into letters, instead of telling it as a continuous narrative in the first person" (1969, p. 18) y Franzel dice que "Hyperion's unique innovation as epistolary novel situates the protagonist's composition of the letters temporally after the conclusion of the events that they narrate, and are thus not simply supposed imprints of emotional states" (2009, p. 160).

15 No vamos a abordar en este caso la verosimilitud literaria de que Hiperión cuente con copias de las cartas que envió o que las recuerde palabra por palabra.
} 
es breve. Tampoco debemos perder de vista que la obra se publicó en dos volúmenes, con dos años de diferencia (1797 y 1799), lo cual indefectiblemente le habrá producido al lector de la época una sensación de tiempo considerable transcurrido entre cartas.

En palabras de Altman, "the temporal aspect of any given epistolary statement is relative to innumerable moments: the actual time that an act described is performed; the moment when it is written down; the respective times that the letter is dispatched, received, read, or reread" (1982, p. 118). Además, la novela epistolar permite la utilización del tiempo a través de las cartas en pos de producir un cierto significado. Lo que afirma Todorov respecto de otra novela también vale para Hiperión: "el hecho de que la novela está narrada en cartas ofrece al relato la posibilidad de deformaciones temporales. Estas deformaciones consisten en el desfase entre el orden cronológico de los acontecimientos descritos y el orden de su sucesión en la novela" (1971, p. 54). Esta característica es funcional a la narración de la Bildung de Hiperión, que, como indicamos, no constituye un relato lineal ni cronológico, sino que está caracterizado por avances y retrocesos y resulta fragmentario.

Otra cuestión fundamental vinculada con el abordaje del tiempo en la novela viene dada por los niveles (y está relacionada, en parte, con lo que señalamos respecto del lector externo y del lector interno y respecto de las brechas temporales) que existen en la novela epistolar, lo que se ha denominado carácter diferido: "el carácter diferido se manifiesta en un doble nivel, es decir, por un lado en el normal de toda comunicación literaria, y por otro, en el nivel originado por el distanciamiento espacio-temporal ficticio entre el remitente y el destinatario de las cartas" (SPANG, 2000, p. 643). Sin duda, esta característica es explotada al máximo por Hölderlin, dado que la obra está dividida en dos volúmenes (separados por dos años de diferencia, lo cual puede otorgarle más verosimilitud a una novela en la que se narran mediante cartas sucesos acaecidos en tierras extranjeras) y, a su vez, cada volumen 
está dividido en dos libros, los cuales a su turno contienen una determinada cantidad de cartas.

Por otro lado, el recurso a la epistolaridad se encuentra justificado y resulta útil y productivo también en virtud del papel preponderante que juegan las cartas en la novela, no ya como parte integrante de la obra literaria, sino como elemento que permite impulsar la acción. Entre otros ejemplos, Belarmino le pide a Hiperión que le cuente su historia (p. 26), Hiperión recibe una carta mientras está redactando otra, por lo cual debe modificarla (p. 148), y es rechazado por su padre mediante una carta (p. 162), algunas cartas de Diotima no llegan (p. 173), Diotima cree que Hiperión murió debido al tiempo entre cartas (p. 192) y, por último, Hiperión se entera de la muerte de Diotima mediante una carta de su amigo Notara (p. 199).

Por fin, corresponde analizar la figura de Belarmino, el destinatario de las cartas de Hiperión. El hecho de que no tengamos ninguna carta proveniente de él es llamativo, y es uno de los motivos que han provocado objeciones a la necesidad de la forma epistolar. Independientemente de que, si hay una carta, esperamos una respuesta en una novela epistolar, creemos que la falta de respuesta no es gratuita ni una "falencia" de la novela.

Algunos autores reducen la importancia de Belarmino en vista de su escasa intervención. Así, Franzel afirma que "the character Bellarmin is absent and so underdeveloped that he is little more than a name/figure" (2009, p. 171) y Silz sostiene que "Bellarmin is completely colorless and unresponsive, a nonentity who adds nothing to the novel or its letter fiction" (1969, p. 17). No obstante, consideramos que, dada la necesaria presencia de un lector interno (Belarmino en este caso) y un lector externo (el lector de la novela) en toda novela epistolar, la ausencia del lector interno le deja lugar al lector externo para que, en cierta medida, lo reemplace. Como apunta Altman, "the external reader's experience is partially governed by the presence of his internal counterpart; we read any given letter from at least three points of view -that of the intended or actual recipient as well as that of the writer and our own" (1982, 
p. 111). De esta forma, si la interpretación del lector interno está ausente, la del lector externo tomará su lugar. Creemos que esta decisión (de prácticamente "borrar" la palabra de Belarmino) es funcional a la Bildung del lector que señaláramos anteriormente, dado que le permite al lector externo ocupar el lugar de Belarmino y sentirse más interpelado aún (más que en una novela no epistolar y más que en una novela epistolar en la que las figuras de remitente y destinatario se intercambian continuamente entre dos o más personajes).

La inclusión del intercambio con Diotima, así como la transcripción de cartas de ella y de las intercambiadas por Notara e Hiperión, le agrega a la novela elementos que no siempre son tenidos en cuenta a la hora de analizar Hiperión en tanto novela epistolar. Si bien Belarmino podría haber tomado el lugar de alguno de estos personajes (pensamos en Notara tal vez) y así asumir un papel más activo y determinante, Belarmino, que es alemán, "no es nadie" justamente para lograr ser todos... los lectores. De tal manera, quedan justificadas la diatriba final y las referencias al público mencionadas antes. La diatriba a los alemanes no es una carta "desvinculada" del resto de la novela, sino la forma de explicitar un destinatario hasta allí implícito.

\section{Conclusión}

Como pudimos apreciar en el análisis que antecede, la forma epistolar de Hiperión se encuentra justificada porque sus características intrínsecas son funcionales al Bildungsroman. En primer lugar, la autoformación que implica todo Bildungsroman se expresa de la mejor manera a través de la intimidad y privacidad que habilita la novela epistolar (género muy popular en el siglo XVIII), especialmente en Hiperión, puesto que la formación del protagonista se vincula estrechamente con su carácter de narrador. El acto de narrar sus vivencias lo ayuda a formarse. En segundo lugar, el elemento temporal que exige la formación de cualquier 
personalidad es compatible con el manejo temporal que habilita la novela epistolar. En Hiperión, la falta de linealidad de la narración (es decir, el hecho de que el narrador no siga un estricto orden cronológico) se ve favorecida por el carácter fragmentario que surge de la división en cartas. ${ }^{16}$ Por último, la pretendida Bildung del lector, elemento frecuentemente analizado en este tipo de novelas y particularmente notorio en Hiperión, en virtud de las expresas interpelaciones al público lector que hemos analizado, es congruente con la necesidad de respuesta y la importancia del destinatario propias de todo intercambio epistolar. Es decir, la elección del género epistolar es la más adecuada para intentar suscitar cierta respuesta en los lectores, pues permite dirigirse expresamente a ellos, en especial cuando el principal destinatario ficcional de las misivas nunca aparece ni toma la palabra, es decir, está ausente.

\section{Referencias bibliográficas}

ALTMAN, Janet. Epistolarity. Approaches to a Form. Columbus: Ohio State University Press, 1982.

COCALIS, Susan. The Transformation of "Bildung" from an Image to an Ideal, Monatshefte, v. 70, n. 4, p. 399-414, invierno de 1978.

DILTHEY, Wilhelm. Vida y poesía. México: Fondo de Cultura Económica, 2016.

FRAANJE, Maarten. The Epistolary Novel in Eighteenth-Century

Russia. Múnich: Sagner, 2001.

FRANZEL, Sean. The Politics and Media of Apostrophe in Hölderlin's Hyperion, The German Quarterly, v. 82, n. 2, p.155-173, primavera de 2009.

FUERST, Norbert. Three German Novels of Education. I. Hölderlin's "Hyperion", Monatshefte, v. 38, n. 6, p. 339-347, octubre de 1946.

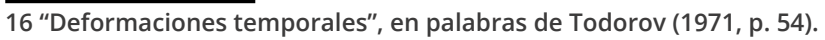


HOFFMEISTER, Gerhart. From Goethe's Wilhelm Meister to anti-Meister Novels: The Romantic Novel between Tieck's William Lovell and Hoffmann's Kater Murr. En MAHONEY, Dennis (ed.). The Literature of German Romanticism. Nueva York: Camden House, 2004. p. 79-99. HÖLDERLIN, Friedrich. Hiperión o el eremita en Grecia. Trad. y pról. de Jesús Munarriz Peralta. Madrid: Ediciones Hiperión, 1996.

HORLACHER, Rebekka. ¿Qué es Bildung? El eterno atractivo de un concepto difuso en la teoría de la educación alemana, Pensamiento Educativo. Revista de Investigación Educacional Latinoamericana, v. 51, n. 1, p. 35-45, 2014.

JOST, François. Variations of a Species: The Bildungsroman,

Symposium: A Quarterly Journal in Modern Literatures, v. 37, n. 2, p. 125-146, diciembre de 2014.

KOVAL, Martín. Vocación y renuncia: la novela de formación alemana entre la Ilustración y la Primera Guerra Mundial. Buenos Aires: Editorial de la Facultad de Filosofía y Letras de la UBA, 2018. MAHONEY, Dennis. The Apprenticeship of the Reader: The Bildungsroman of the "Age of Goethe". En HARDIN, James (ed.).

Reflection and Action: essays on the Bildungsroman. Columbia: University of South Carolina Press, 1991, p. 97-117.

MILES, David. The Picaro's Journey to the Confessional: The Changing Image of the Hero in the German Bildungsroman, Modern Language Association, v. 89, n. 5, p. 980-992, 1974.

NAUEN, Franz. Revolution, Idealism, and Human Freedom: Schelling, Hölderlin, and Hegel and The Crisis of Early German Idealism. La Haya: Martinus Nijhoff, 1971.

REDFIELD, Marc. The Phantom Bildungsroman. En Phantom Formations. Aesthetic Ideology and the Bildungsroman. Ithaca: Cornell University Press, 1996, p. 38-62.

SAMMONS, Jeffrey. The Bildungsroman for Nonspecialists: An Attempt at a Clarification. En HARDIN, James (ed.). Reflection and Action:

Essays on the Bildungsroman. Columbia: University of South Carolina Press, 1991, p. 26-45. 
Hiperión como novela epistolar

Juan Pau • Mariela Ferrari

SILZ, Walter. Hölderlin's Hyperion: a critical reading. Filadelfia: University of Pennsylvania Press, 1969.

SPANG, Kurt. La novela epistolar. Un intento de definición genérica, RILCE: Revista de Filología Hispánica, v. 16, n. 3, p. 639-656, 2000.

STEPHENSON, Roger. The Novel in Weimar Classicism: Symbolic Form and Symbolic Pregnance. En RICHTER, Simon (ed.). The Literature of Weimar Classicism. Rochester: Camden House, 2005, p. 211-235.

SWALES, Martin. Irony and the Novel: Reflections on the German Bildungsroman. En HARDIN, James (ed.). Reflection and Action: Essays on the Bildungsroman. Columbia: University of South Carolina Press, 1991, p. 46-68.

TODOROV, Tzvetan. Literatura y significación. Barcelona: Editorial Planeta, 1971. 